\title{
ACTUACIÓN DIDÁCTICA DE LOS DOCENTES DE LA UNIVERSIDAD ESTATAL PENÍNSULA DE SANTA ELENA. RESULTADOS DE UNA EXPERIENCIA DE COEVALUACIÓN
}

\section{DIDACTIC PERFORMANCE OF THE TEACHERS OF THE PENÍNSULA DE SANTA ELENA STATE UNIVERSITY. RESULTS OF A COEVALUATION EXPERIENCE}

\author{
María Caridad Mederos Machado ${ }^{1^{*}}$ \\ Carlos Eloy Balmaseda Espinosa ${ }^{1}$ \\ Marilin Balmaseda Mederos ${ }^{2}$ \\ Lilia Valencia Cruzatti ${ }^{1}$
}

1Universidad Peninsular de Santa Elena, Ecuador. “mmederos@upse.edu.ec ${ }^{2}$ Universidad Católica de Cuenca, Ecuador

\section{RESUMEN}

El ejercicio de la cátedra y la investigación bajo la más amplia libertad sin ningún tipo de imposición o restricción religiosa, política, partidista o de otra índole está reconocida en la Constitución de Ecuador y en otras normativas que establecen los derechos y obligaciones de los docentes. El hecho de que en el país exista libertad de cátedra no es incompatible con la idea de que en las clases de las universidades se observen principios y procedimientos metodológicos, que consideren diferentes corrientes y teorías relacionadas con el aprendizaje como las que indica Larrea (2014) con el análisis de la complejidad sistémica aplicada al currículo universitario, el Constructivismo de (Ausubel, 1991), la teoría de la determinación social del desarrollo psíquico (Vigotsky, 1978), la teoría de la complejidad (Morín, 1999), el Conectivismo (Siemens, 2004), Pedagogía crítica (Freire, Faundez, 2010), Formación en competencias (Posada, 2011), entre otras. También relacionadas con la formación de valores, que deben ser atendidos dentro del proceso docente. El presente trabajo tiene como objetivo analizar los aciertos y desaciertos didácticos de los profesores de la Universidad Estatal Península de Santa Elena (UPSE), a partir del procesamiento de los instrumentos utilizados para la evaluación de pares, en un período académico, (una guía de observación de respuestas dicotómicas). El trabajo examina esta guía aplicada a todos los docentes evaluados, a partir de un análisis de frecuencia en cada ítem estudiado. Los resultados alcanzados permiten diseñar acciones metodológicas basadas algunas en el Modelo lceberg (De Miguel, 2005) y en los autores ya citados, a nivel institucional para orientar mejor la formación del docente en el propio puesto de trabajo. Se concluye que los principales problemas detectados se relacionan con el estímulo a la investigación y a la lectura y a la necesidad deponer énfasis en el aprendizaje interactivo y un uso adecuado de los medios.

Palabras clave: Didáctica, docentes, métodos de enseñanza- aprendizaje

\begin{abstract}
The exercise of academic and research under the broadest freedom without any imposition or religious, political, partisan or other restrictions it is recognized in the Constitution of Ecuador and other regulations that establish the rights and obligations of teachers. The fact that there is academic freedom in the country is not incompatible with the idea that in the classes are observed universities principles and methodological procedures that consider different trends and theories related to learning like as Constructivism (Ausubel, Novak, Hanesian, 1978), the theory of social determination of mental development (Vygotsky, 1978), the theory of complexity (Morin, 1999), Connectivism (Siemens, 2004), critical pedagogy (Freire, Faundez, 2010), training in skills (Posada, 2011), among others. Also related to the formation of values, which must be addressed within the teaching process. This paper aims to analyze the successes and failures of teachers of the UPSE, from processing of the instruments used for peer review in an academic period. Peer assessment forms applied to all teachers evaluated from a frequency analysis on each item studied are discussed. The results allow some design methodological actions based on the Iceberg Model (De Miguel, 2005) and the authors already mentioned, at the
\end{abstract}


institutional level to better target the training of teachers in the job itself. It is concluded that the main problems relate to the encouragement of research and reading and to the need to depose emphasis on interactive learning and appropriate use of the media.

Key words: Teaching, teachers, teaching and learning methods.

Recibido: 12 de marzo de 2018

Aceptado: 12 de junio de 2018

Publicado: 20 de junio de 2018

\section{Marco teórico}

En las consideraciones de Larrea (2014) acerca de la formación e integración del personal académico en las universidades ecuatorianas se refiere a que "los docentes de las universidades en su mayoría no cumplen con los niveles de cualificación y titulación que la Ley Orgánica de la Educación Superior (LOES) exige y que el proceso de formación de los docentes para el cumplimiento de los nuevos perfiles y funciones que las IES necesitan, no está lo suficientemente consolidado, se invierten recursos en cursos con paradigmas desactualizados y centrados fundamentalmente en metodologías didácticas, que difieren de los parámetros de desempeño planteados por la LOES y sus normativas".

Esta situación tiene por lo tanto un reflejo en el comportamiento didáctico de los profesores a la hora de impartir sus materias y de desarrollar el currículo para los que trabajan en las universidades.

En la actualidad, en los circuitos académicos se apela con insistencia a una ruptura paradigmática en el modelo educativo de las universidades y de la educación superior. La reforma en que se encuentra la universidad ecuatoriana actual lleva la impronta de lo que Morin (1999) identifica como "revolución paradigmática" o la llamada también, "reforma del pensamiento". Existen definiciones derivadas de las diferentes teorías del aprendizaje y tendencias pedagógicas contemporáneas, que dan respuesta a esta pregunta. Desde esta perspectiva de análisis, pueden señalarse aquellas que pertenecen a grandes figuras mundiales que se han dedicado a su estudio, y por tanto son clásicas en el tema, a continuación se exponen algunas de ellas:

Según Vigotski (1978) "El aprendizaje es una actividad social, y no solo un proceso de realización individual como hasta el momento se había sostenido; una actividad de producción y reproducción del conocimiento mediante la cual el alumno asimila los modos sociales de actividad y de interacción, y más tarde en la escuela, subyacen, los fundamentos del conocimiento científico, bajo condiciones de orientación e interacción social."

Ausubel (1991) introduce el concepto de Aprendizaje significativo. "Aprender es una adquisición permanente de cuerpos estables de conocimientos y de las capacidades necesarias para adquirir tales conocimientos."

Para el autor este proceso está condicionado por varios factores, entre ellos por la significatividad lógica y psicológica del material de aprendizaje y por la disposición del sujeto que aprende.

Al mismo tiempo dichos factores sólo se dan si el nuevo contenido de aprendizaje cobra nuevo significado para el estudiante. Ello quiere decir que para poder contrastar los nuevos contenidos, el sujeto recurre a sus ideas previas sobre el tema en cuestión y trata de contrastar los aprendizajes con ellas.

Según este autor, en la medida en que los conocimientos que se poseen sean más disonantes con los nuevos, resultarán más significativos y entonces habrá mayores posibilidades de que cobren significado $y$ se integren al sistema de conocimientos construidos.

La descripción de las relaciones internas entre el pensamiento y el lenguaje es otro aporte de Vigotsky al desarrollo de las ciencias psicológicas y a las teorías que sustentan el proceso de aprendizaje. Al considerar el papel del lenguaje como modo de representación de la realidad y al mismo tiempo como la forma de concretar y expresar las adquisiciones fundamentales de las personas en el devenir de su pensamiento, se puntualiza en su función de representación y al mismo tiempo de medio de comunicación y emisión de mensajes.

Por ello es importante que los profesores conozcan y profundicen no solo en las ideas previas de los estudiantes, sino en las teorías implícitas de los mismos para poder conducir de manera más eficiente el proceso de construcción de conocimientos.

La Didáctica es considerada una rama de la Pedagogía que se encarga de estudiar los componentes de los procesos de enseñanza y aprendizaje que facilitan la apropiación o construcción de conocimientos, habilidades y actitudes. No es exclusiva de la institución educativa, pues la misma siempre se enriquece desde las más disímiles experiencias de aprendizaje. El objeto de estudio de la Didáctica se ha ido ampliando 
y en la actualidad se ocupa, en el ámbito institucional, del estudio de la clase como forma organizativa, objetivos, contenidos, métodos, medios, la evaluación, los sujetos que aprenden y los entornos de aprendizaje. No como recetas ni un conjunto de reglas, sino vistas como un conjunto de ideas y conceptos que garantizan coherencia y entendimiento en el acto de aprender. El proceso de aprendizaje ha sido estudiado en profundidad, desde hace décadas.

Al analizar la Gestión Curricular de la educación superior Técnica y Tecnológica y de grado en Ecuador, Larrea (2014) se refiere a "Modelos pedagógicos con énfasis en contenidos repetitivos, irrelevantes, de "oficio", de respuesta directa a los vaivenes del mercado, que dejan de lado al sujeto educativo y la integralidad de sus aprendizajes. La organización de los aprendizajes no es una prioridad, en la mayoría de las veces el aula es el único ambiente de aprendizaje, insuficiencia de modelos de formación ciudadana y de educación intercultural".

El Art. 29 de la Carta Magna ecuatoriana señala que el Estado garantizará la libertad de enseñanza, la libertad de cátedra en la educación superior, y el derecho de las personas de aprender en su propia lengua y ámbito cultural. La LOES (2010) en el acápite Derechos de los profesores o profesoras e investigadores o investigadoras refiere: "Son derechos de los profesores $\mathrm{o}$ profesoras $\mathrm{e}$ investigadores o investigadoras de conformidad con la Constitución y esta Ley los siguientes:

Ejercer la cátedra y la investigación bajo la más amplia libertad sin ningún tipo de imposición o restricción religiosa, política, partidista o de otra índole..."

Esta garantía es pleno ejercicio de su autonomía responsable, entendida como la facultad de la institución y sus profesores para exponer, con la orientación y herramientas pedagógicas que estimaren más adecuadas, los contenidos definidos en los programas de estudio.

Por lo tanto se garantiza la libertad investigativa, comprendida como la facultad de la entidad y sus investigadores de buscar la verdad en los distintos ámbitos, sin ningún tipo de impedimento $\mathrm{u}$ obstáculo, salvo lo establecido en la Constitución y en la presente Ley.

A menudo se confunde la libertad de cátedra con un falso beneficio que profesa: el docente es libre de hacer o decir lo que quiera y como quiera. Pero la libertad de cátedra está sujeta a ciertos límites. Se trata de un derecho del docente recogido en la Constitución y que en su origen en Europa protegía al profesorado frente a la censura del Estado, permitiendo que la enseñanza no estuviera dirigida por las doctrinas morales que regían en política.
El sentido último de esta libertad es reconocer el derecho a orientar su enseñanza de la manera que considere más conveniente en función de sus investigaciones y de las teorías científicas. De modo que la libertad de cátedra evita que se coarte la amplitud de enfoques y la formación de un criterio propio por los alumnos del nivel universitario. Vista de esta forma, no resulta contradictorio que se observen determinados principios en el proceso de enseñanza aprendizaje.

Como forma de actuación se identifican diferentes sistemas de principios didácticos, Silvestre y Zilberstein (2000); Labarrere y Valdivia (1988); Zankov (1975).

Según Labarrere y Valdivia (1988) los principios didácticos incluyen tanto la acción de los docentes como la de los estudiantes. Las autoras reconocen como principios:

- Del carácter científico de la enseñanza.

- Del respeto a la clase como principal forma organizativa en la institución educativa.

- De la sistematización de la enseñanza.

- Del carácter educativo de la enseñanza.

- La planificación del aprendizaje como parte del desarrollo del estudiante.

- De la asequibilidad. Los contenidos deben ser trabajados desde los niveles más simples del conocimiento a los más complejos.

- Los objetivos o resultados de aprendizaje deben constituir la guía de las decisiones que se tomen en el proceso de enseñanza aprendizaje.

- De la relación entre la teoría y la práctica.

- Del carácter consciente y activo de los alumnos bajo la guía del profesor.

- De la solidez de la construcción de los conocimientos, habilidades y hábitos.

- De la atención a las diferencias individuales dentro del carácter colectivo del proceso docente - educativo.

- Del carácter audiovisual de la enseñanza: unión de lo concreto y lo abstracto".

- Del diagnóstico integral de la preparación del alumno para las exigencias del proceso de enseñanza aprendizaje, y seguimiento de sus logros.

Es precisamente este sistema de principios didácticos lo que sirve de base a esta investigación. Al mismo tiempo que la didáctica se encarga del proceso de enseñanza de las ciencias y de las asignaturas del currículo, se desarrolla en un contexto educativo determinado, donde se forman valores. El valor no se aprende desde la lección teórica, sino que se construye en la experiencia vivida. Molina et al. (2008) refieren que los valores que deben fomentarse en la educación superior son: amistad, creatividad, libertad, alegría, autoestima, igualdad, humildad, respeto, dignidad, sensibilidad. Los autores de este trabajo consideran que si bien 
esos valores son deseables, es importante en el ámbito universitario reforzar los que a continuación se describen.

Según Moreno (2011) la educación superior tiene su propia concreción, por tanto, requiere una didáctica distintiva que posibilite el aprendizaje de los alumnos, en su mayoría adultos, con conocimientos y experiencias previas, motivaciones $\mathrm{y}$ expectativas diversas respecto a su proyecto personal y profesional. Aunque existen distintos enfoques y propuestas didácticas hay ciertos planteamientos que parecen más acordes con las exigencias que se plantean actualmente a las instituciones de educación superior en todo el mundo.

En el presente de la educación superior ecuatoriana resulta imprescindible manejar los documentos rectores para la planificación del proceso de aprendizaje. Unos provienen directamente de las normativas (Reglamento de régimen académico, Diseños curriculares) pero otros (Sílabos, Planes de clases, Aulas virtuales) son elaborados por los profesores, los que a su vez tienen su expresión en las clases que se imparten.

Las instituciones de educación superior están llamadas a garantizar la calidad de la educación y la enseñanza- aprendizaje, en sus centros, por lo que la actuación didáctica de los profesores se considera un indicador que es importante evaluar de forma continua por pares, los que a su vez se reconocen en los aciertos y errores de sus colegas. Al mismo tiempo constituye un elemento para la evaluación del desempeño docente.

Un primer eje a considerar, según Gimeno (1998), citado por Vain (1998) es "sugerir tres instancias en el desarrollo de la Evaluación de la Docencia Universitaria:

1. La docencia tomada como un componente de la función enseñanza que requiere ser visualizada por la institución, a los fines de mejorar su calidad. Estamos pensando en un aspecto a ser analizado en los procesos de autoevaluación institucional y evaluación externa de las instituciones de educación superior.

2. La docencia estudiada como aspecto de las políticas institucionales trazadas para el desarrollo de los recursos humanos. Estamos ubicando la evaluación en relación a la implementación de la Carrera Docente y por lo tanto situada en el marco de procesos como el Ingreso, Formación, Permanencia, Promoción, etc.

3. La docencia integrada a la actividad cotidiana de la enseñanza, en cuanto actividad que es desarrollada por determinados actores, en tiempos y espacios específicos, con ciertos efectos y resultados. Estamos apuntando a la evaluación que, operando al interior de la cátedra, puede contribuir a mejorar la enseñanza".

El conectivismo, según la RAE (2010) tiene sus principios, que se deben entender de múltiples maneras, en primer lugar como "las proposiciones o verdades fundamentales por donde se estudian las ciencias o las artes y las normas fundamentales que rigen el pensamiento y la conducta".

Es posible identificar algunos principios propuestos o interpretados por Siemens (2004) y otros seguidores. Por lo abundante de la información que existe en este sentido se mencionan a continuación algunos principios sin anexar una fuente concreta.

- El aprendizaje y el conocimiento yacen en la diversidad de opiniones.

- Es necesario nutrir y mantener las conexiones para facilitar el aprendizaje continuo

- La habilidad para ver las conexiones entre los campos, ideas y conceptos es primordial.

- La información actualizada y precisa es la intención de todas las actividades del proceso conectivista.

- La toma de decisiones es en sí misma un proceso de aprendizaje. Escoger qué aprender y el significado de la información entrante es visto a través de la lente de una realidad cambiante.

- La integración de la cognición y las emociones en las decisiones es importante. El pensamiento y las emociones se influyen mutuamente.

- El aprendizaje es un proceso de conectar nodos o fuentes de información especializada. Un estudiante puede mejorar exponencialmente su propio aprendizaje si se conecta con otras redes existentes.

- El aprendizaje puede residir en dispositivos no humanos, puede estar también en una comunidad, una red o una base de datos.

Este trabajo tiene como objetivo analizar los principales elementos didácticos que se manifiestan en la actividad docente de los profesores de la UPSE.

\section{Método}

Se utilizaron métodos teóricos para el análisis documental que sustenta este trabajo, como el análisis y la síntesis. Los mismos hicieron posible resumir las diferentes visiones acerca de la didáctica, sus principios y valores relacionados.

Como método empírico se utilizó el instrumento que se usa para la evaluación áulica por pares entre docentes de la UPSE.

Se estudió el $98 \%$ del claustro de docentes de la UPSE. Para ello se analizaron 494 evaluaciones de pares, tanto en su generalidad como en los casos específicos de las facultades. Para analizar los datos 
obtenidos en la evaluación áulica, en este trabajo se toman como referencia los resultados generales de la universidad y las Facultades de Ciencias Agrarias, Ciencias Administrativas, Ciencias Sociales y de la Salud, y Ciencias de la Educación e Idiomas, en función de la cantidad de profesores y atendiendo a su pertinencia con este tema específico, como es el caso de la facultad de Ciencias de la educación e idiomas.

El procesamiento se realizó utilizando el programa Excel de Microsoft office.

\section{Análisis de datos}

Según Contreras (2016) "en los profesores existe un conjunto frecuente de creencias y creencias de actuación curricular, con respecto a qué enseñar (contenidos), cómo enseñar (metodología) y, qué y cómo evaluar (evaluación)". De modo que en referencia a todos estos aspectos se evidencia que son observados por los docentes evaluados. El cómo enseñar es complejo para dichos profesores.

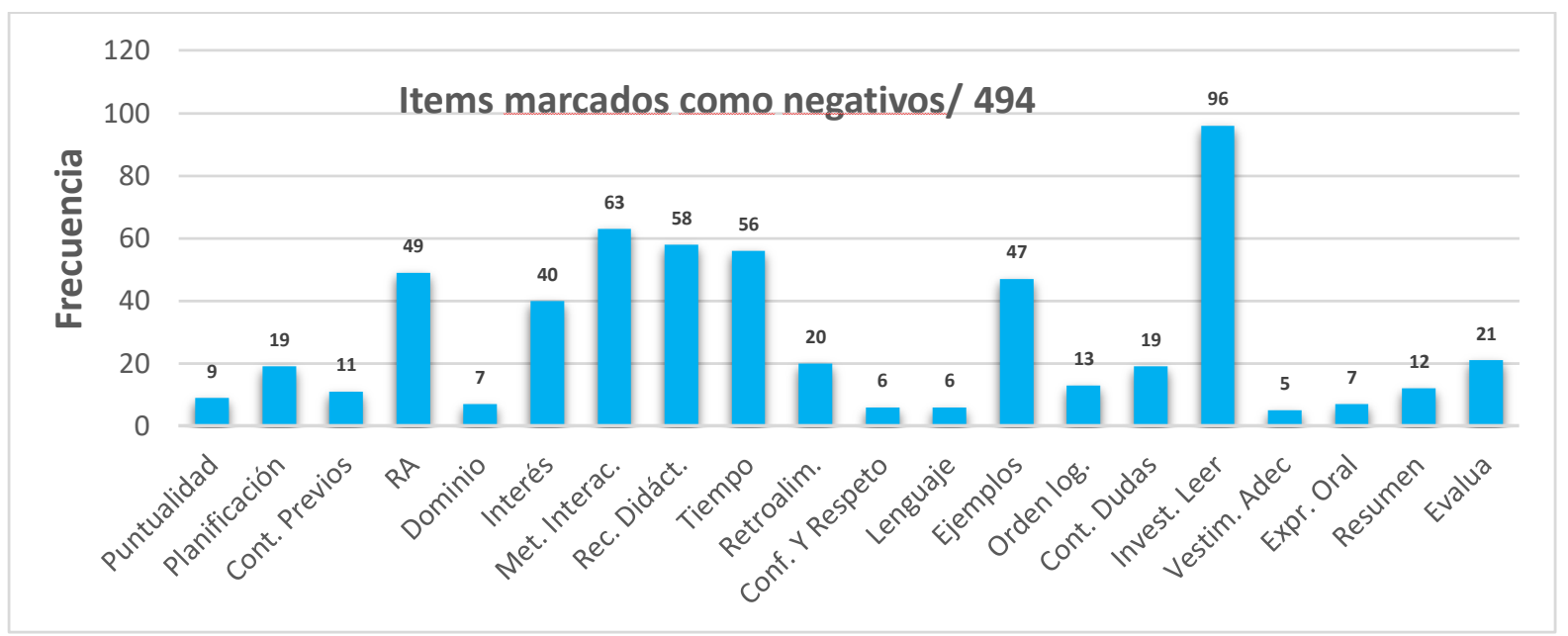

Gráfico 1. Resultados generales UPSE. Evaluación áulica.

Fuente: Elaboración propia de los autores.

Al analizar el gráfico 1, se observa que la principal dificultad se encuentra en el estímulo a la lectura y a la investigación, seguido de la necesidad de incrementar los métodos interactivos y en el uso de los recursos didácticos y el tiempo.

La investigación señala que en relación con el dominio de los contenidos son escasas las dificultades, coincide con los hallazgos de Contreras (2016) en que en el "aspecto curricular de contenidos los profesores creen importante el dominio conceptual según la lógica de la disciplina, resaltando el método científico". Ello pone de manifiesto las epistemologías de los profesores, con respecto a la naturaleza del contenido escolar. Sin embargo, una minoría da importancia a los contenidos procedimentales Esta tendencia más fragmentada, también se ha encontrado respecto al uso de ejemplos de la vida cotidiana como contenidos para establecer relaciones y explicar. Lo anterior, se relaciona con aspectos importantes del conocimiento y pensamiento sobre el contenido curricular a enseñar, como lo son su selección, su secuenciación y su organización.

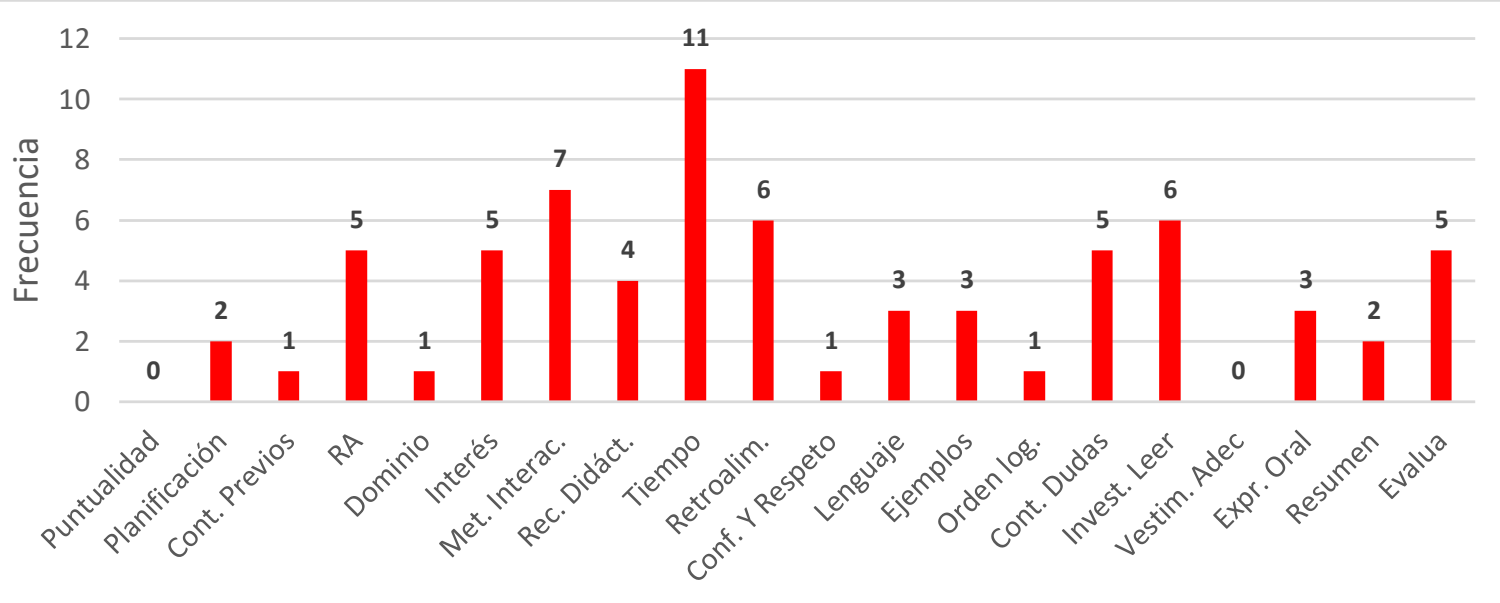

Gráfico 2. Ciencias Sociales y de la Salud/59 
Fuente: Elaboración propia de los autores.

En el gráfico 2 se aprecia que la dificultad principal en la Facultad de Ciencias Sociales y de la Salud se concentra en el uso racional del tiempo, en el uso de métodos interactivos, en retroalimentar el contenido y en el estímulo a la investigación y la lectura.

Se señalan como elementos positivos el dominio de la ciencia y el uso del lenguaje, aunque es preciso retomar autores $\mathrm{y}$ hechos de la ciencia contemporánea. Ante esta situación se potencia la actualización continua de conocimientos por parte de los profesores, para poder brindar una educación de calidad donde los actuales dominios de las ciencias estén reflejados.
También la evaluación es un elemento importante para la superación de los profesores evaluados en esta Facultad.

Estos resultados coinciden con los de la investigación desarrollada por González (2013) en profesores de Ciencias Sociales de Educación Media, en la que sus dificultades principales corresponden al área metodológica. A diferencia de los profesores de las Ciencias Exactas y Técnicas, que como en esta investigación (Gráfico 3) la distribución del tiempo es de las variables menos controladas por los docentes.

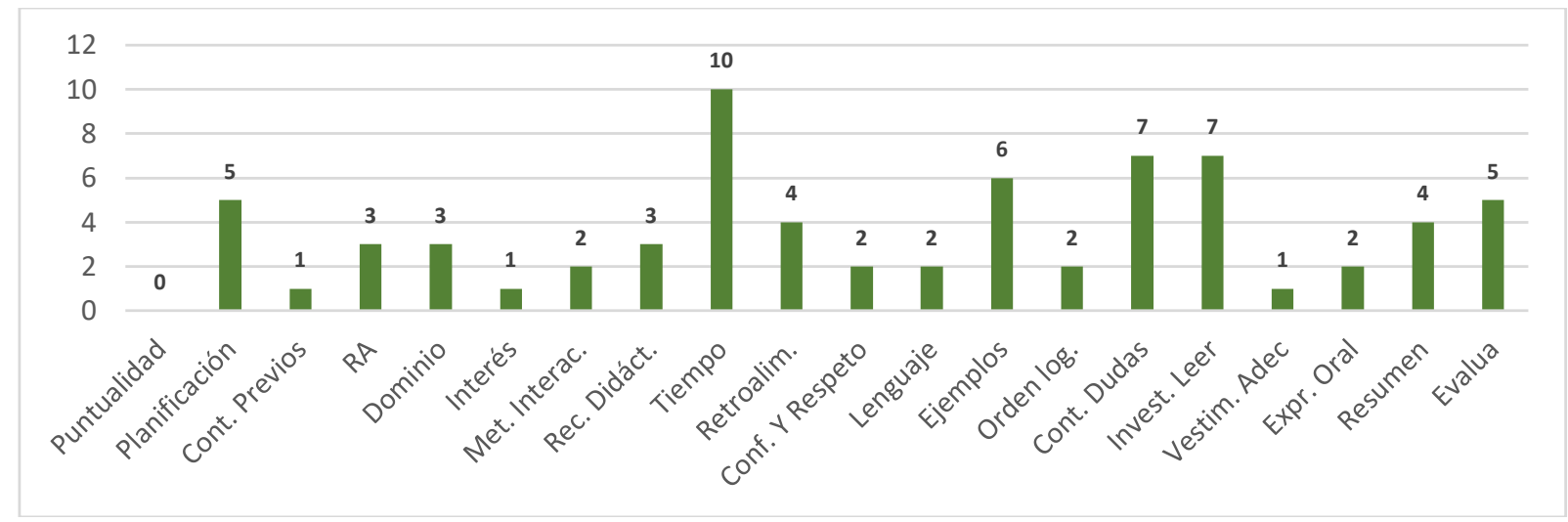

Gráfico 3. Ciencias Agrarias/48

Fuente: Elaboración propia de los autores.

En la Facultad de Ciencias Agrarias (Gráfico 3) se destaca como dificultad el uso del tiempo, seguidos por la escasa vinculación del contenido con el contexto y el poco estímulo a la investigación y la lectura.

La Facultad de Ciencias Administrativas (Gráfico 4) es la mayor y la que más docentes cuenta en su planta. El uso de recursos didácticos, de métodos interactivos, la orientación del contenido hacia el resultado de aprendizaje y el estímulo a la investigación y a la lectura, son las principales dificultades que aparecen en estos docentes.

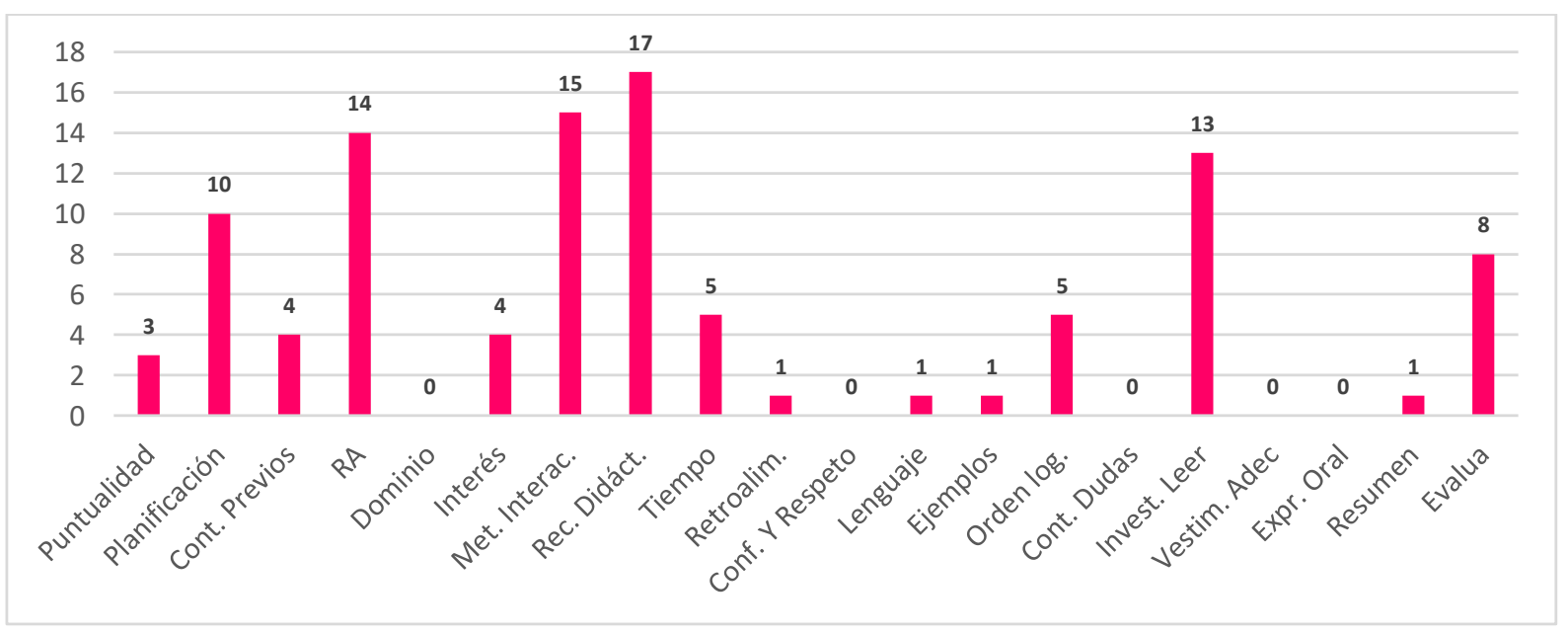

Gráfico 4. Ciencias Administrativas/150

Fuente: Elaboración propia de los autores. 
En el gráfico 5 se puede observar que en los docentes de la Facultad de Ciencias de la Educación e Idiomas se concentran las dificultades en el estímulo a la lectura y la investigación así como en la orientación del contenido hacia el resultado de aprendizaje que se planifica. En la investigación de González (2013) los profesores de Ciencias Sociales y Pedagogía, muestran un comportamiento similar.

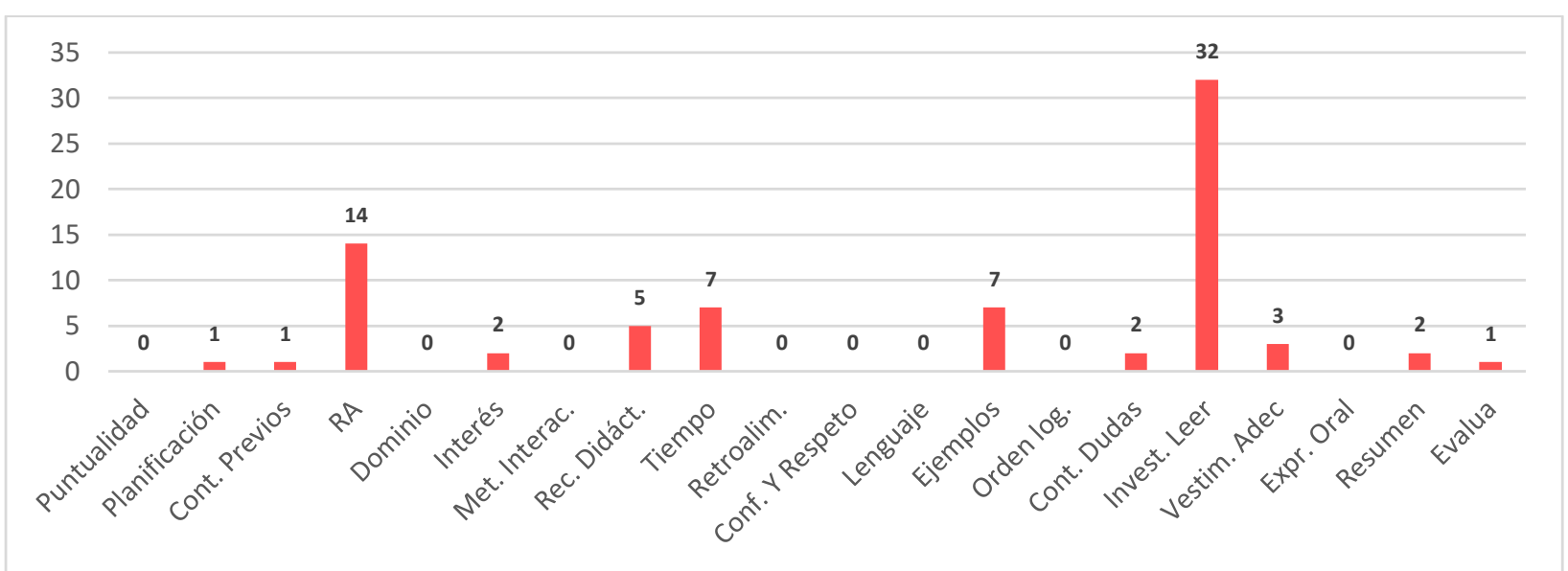

Gráfico 5. Ciencias de la Educación e Idiomas/65

Fuente: Elaboración propia de los autores.

\section{Discusión y Conclusión}

Las dificultades más comunes están relacionadas con la capacidad didáctica de los docentes para promover el interés de los estudiantes por la investigación científica y por las lecturas. El uso racional del tiempo durante la clase es otro elemento que se manifiesta, dando lugar a que contenidos interesantes y necesarios sean abordados con muy poco tiempo y otros susceptibles de ser desarrollados desde el trabajo autónomo se les dedica más tiempo del que requiere en una actividad asistida por el profesor.

Orientar el contenido de las asignaturas mediante la explicación clara y oportuna del resultado de aprendizaje de la clase es otro elemento que no es atendido suficientemente.

Por otro lado, y aunque la investigación ha considerado profesores de las diversas especialidades y trayectorias de experiencia profesional, tanto la evidencia cuantitativa como cualitativa, señala que no existen diferencias marcadas entre las distintas disciplinas o diferentes niveles de enseñanza.

Los resultados de esta experiencia de coevaluación sirvieron de base diagnóstica para desarrollar un curso taller para docentes de la UPSE sobre Didáctica de la Educación Superior.

\section{Bibliografía}

Ausubel, D. (1991).Teoría del Aprendizaje significativo. Paidós. España.
Contreras, S. (2016) Pensamiento Pedagógico en la Enseñanza de las Ciencias. Análisis de las Creencias Curriculares y sus Implicancias para la Formación de Profesores de Enseñanza Media En Revista Formación Universitaria. Vol. 9(1), 15-24 (2016). Disponible en http://www.scielo.cl/pdf/ formuniv/v9n1/art03.pdf

Gimeno S, J. El curriculum: una reflexión sobre la práctica. Editorial Morata. Madrid, 1988.

González, I. (2013) Estudio de las prácticas didáctico- metodológicas de los profesores de ciencias sociales y de Pedagogía del Instituto Pedagógico Piura. En Cuaderno pedagógico. Separata 3. Año 5.

Labarrere. G. y Valdivia G., Pedagogía, 1988, p. 56

Larrea, E. (2014) El currículo de la educación superior desde la complejidad sistémica. Documento interno como Guía del CES para rediseños curriculares.

Molina, Luzcarin, Pérez Pellín, Siulbel, Suárez, Auxifrantys, \& Rodríguez G, William A. (2008). La importancia de formar en valores en la educación superior. Acta Odontológica Venezolana, 46(1), 41-51.

Moreno, T. (2011) Didáctica de la Educación Superior: nuevos desafíos en el siglo XXI.Universidad Autónoma Metropolitana. Unidad-Cuajimalpa. México.

Morín E. (1999). L'intelligence de la complexité, editado por Le Harmattan, París 1999. Traducción de Jose Luis Solana Ruiz.

RAE. Versión en línea de la obra de la Real academia de la lengua española. Disponible en 
http://dle.rae.es/?w=diccionario. Consultada el 13 de junio de 2016.

Siemens, G. (2004). Conectivismo: Una teoría de aprendizaje para la era digital.

Silvestre, M y J, Zilberstein, Enseñanza y aprendizaje desarrollador, 2000, p. 22

Vain, P. (1998) La evaluación de la docencia universitaria: un problema complejo. Documento presentado en el marco de la Convocatoria organizada por CONEAU para la realización de trabajos Teórico-Metodológicos sobre evaluación institucional universitaria. Universidad Nacional de Misiones. Argentina, p.6

Vigotsky, L. S. (1978). Pensamiento y lenguaje, Madrid. Editorial Paidós.

Zankov, L, La enseñanza y el desarrollo, 1975, p. 70. 